\title{
DAS RELIEF DER ERDE
}

\section{E. K. GeRbER}

In diesem Jahr ist in zweiter umgearbeiteter Auflage das 1100 Seiten umfassende Werk "Das Relief der Erde " ${ }^{1}$ von FriTz MachatscheK erschienen, das 1940 zum ersten Mal aufgelegt wurde. Es sei mir als "seinem einzigen Schweizerschüler" während seiner Dozentur an der ETH Zürich 1924-28 gestattet, eine persönliche Erinnerung vorauszuschicken. Mit größter Regelmäßigkeit ist der damals 50 Jährige, in der Hand die Arbeitsmappe, ins Institut gekommen, wo er ausdauernd, ungestört von Lärm und Ablenkung eines großen Betriebes, eifrigen Literaturstudien oblag. Heute, kurz vor seinem 80 . Geburtstag liegt nun die 2. Auflage seines Werkes vor, einer Lebensarbeit, denn solche Werke, deren Inhalt sich auf der Kenntnis von tausenden von Schriften aufbaut, setzt nicht nur vorübergehende Beschäftigung mit dem Gegenstand voraus, sondern stützt sich neben jahrzehntelangem Fleiß auf eine ganz besondere Begabung der Verarbeitung und Einordnung, besonders auch der kritischen Sichtung. Diese Begabung MachatscheKs hat sich genau vor einem halben Jahrhundert in seinem Werk "Der Schweizer Jura geäußert, einer Arbeit, die trotz der zeitbedingten Veralterung doch ein zusammenfassendes Standardwerk geblieben ist, das keine Juraforschung übersehen darf. Es ist klar, daß bei einer erdumspannenden Arbeit der Autor zum größten Teil auf die Zuverlässigkeit fremder Forscher angewiesen ist, die Arbeit also vorwiegend referierend, aber doch kritisch auswählend und abwägend sein muß. Man bewundert durch das ganze Werk das Geschick, mit dem Маснатьснек diese Aufgabe gelöst hat. Es ist klar, daß einem solchen Werk auch Ungenauigkeiten anhaften und Lücken vorhanden sind, daß auch der Verfasser die beängstigend angeschwollene Spezialliteratur der letzten Jahre kaum mehr restlos bewältigen konnte.

Diesen von einem Einzelnen nicht vermeidbaren Mängeln allzu großes Gewicht beizulegen, heißt allen Bestrebungen eines Einzelnen, einen globalen Überblick vorzulegen, die Berechtigung absprechen. Es ist aber zu hoffen, daß trotz aller Schwierigkeiten immer wieder einzelne Gelehrte die Aufgabe übernehmen, eine weltweite Schau eines ganzen Gebietes vorzulegen, denn ein solcher Überblick ist ein Markstein des Wissens einer bestimmten Zeit, eine Standortsbestimmung.

Der ganze erste Band und ein Achtel des zweiten sind dem eurasischen Kontinentalblock (Britisches Schollen- und Stufenland, Französisch-mitteleuropäisches Schollen- und Stufenland, Fennoskandia, Osteuropa, Sibirien, Zentralasien, Ostasien) und dem mediterranen Gebirgsgürtel der alten Welt (Atlas, Iberien, Korsardinien, Alpen, Jura und Alpenvorland, Karpaten, Balkan, Krim, Pontus) gewidmet, davon gute ${ }^{4} / 5$ europäischen Gebieten. Der zweite Band umfaßt (neben Anatolien, Armenien, Kaukasien, Iran, Himalaya, Hinterindien, Malayischer Archipel) im weitern das altweltliche Gondwanaland (Afrikanische Masse, Vorderasiatische Schollen, Dekan und Ceylon, Australische Masse, Indik), Ozeanien, die Neue Welt (Laurentischer Rumpf, Appalachen, Küstenebenen, Tiefländer, Kordilleren, Mittelamerika, Anden, außerandines Südamerika), den atlantischen Ozean und Antarktika. Wenn auch wohl in einem amerikanischen Werk die Neue Welt ausführlicher zur Sprache käme, so entspricht doch dieses Überwiegen Europas weitgehend unsern tatsächlichen Kenntnissen. Wir sind noch weit entfernt von einer "europäischen" Durchforschung der ganzen Erde.

Aus der Großeinteilung der Werkes geht hervor, daß MachatscheK nicht den üblichen Erdteilen folgt, sondern morphotektonische Einheiten (Gondwanaland!) zu Grunde legt. Dieser Leitgedanke morphotektonischer Gliederung wird konsequent bis zu den kleinsten Formeinheiten durchgeführt. Den einzelnen Abschnitten geht meist eine Einleitung über den geologischen Bau und die Bildungsgeschichte voraus. Die Morphogenese, die Chronologie der Vorgänge steht im Vordergrund. Auf diese Betrachtungsweise sind auch die Illustrationen ausgerichtet. Im ganzen Werk finden wir nur 2 Blockdiagramme, dafür über hundert morphologische Karten mit meist stark geologisch-tektonischem Einschlag und gut hundert geologische Profile. Wenn wir von einer Standortsbestimmung sprachen, so ist natürlich nicht zu übersehen, daß sich in der notwendigen Auslese des Stoffes die Persönlichkeit des Autors spiegelt. Dies zeigt sich hier im stark regional-geologischen Einschlag, auch in einem Zug zum abstrakten Darstellen. Bei so zeichnerisch begabten Morphologen wie DE MARTonne oder Davis würde nicht die Karte und das Querprofil, sondern die Geländeansicht und das Blockdiagramm, d. h. der Versuch der direkten Formbetrachtung, der anschaulichen Dreidimensionalität, vorherrschen. Umso mehr widmete sich MaCHAтscheк dem Referieren einer sehr ausgedehnten Literatur. Es lassen sich drei Betrachtungsarten des Reliefs unterscheiden. Das erste Stadium ist das Studium der orographischen Verhältnisse. Noch in der Generation vor MACHATSCHEK haben sich führende Morphologen - Geographen wie Geologen - mit rein orographischen Fragen beschäftigt. Verteilung von Gebirge und Flachland, Gebirgstypen, Anordnungsprinzipien waren das Ziel der Forschung. Die Verbesserung der topographischen Karte hat merkwürdigerweise die Entwicklung einer Stilkunde des Reliefs nicht gefördert, sondern auf Nebengeleise geführt, wie die morphometrischen Versuche zeigen, die nie globale Bedeutung erhielten, da sie meist zu unanschaulichen Zahlenwerten führen. Von dieser morphologischen Vorphase finden

1 Machatschek, Fritz: Das Relief der Erde. Versuch einer regionalen Morphologie der Erdoberfläche. Band I: 531 Seiten, 10 Tafeln und 142 Textfiguren. Band II : 594 Seiten, 10 Tafeln und 186 Textfiguren. Gebrüder Bornträger. Berlin 1955. 
wir in Maснатscheк nur Rudimente, so * Das Gebiet der meridionalen Stromfurchen * (Band II) eine fast rein orographische Beschreibung.

Die zweite Stufe, die die orographische Erforschung roraussetzt, ist die geologisch-tektonische Betrachtung, die den Stil des Reliefs aus dem Innern, aus dem Bau, begründet. Diese Stufe kann sich schon auf ein Standardwerk der letzten Generation, auf ED. SuEss' Antlitz der Erde, stützen. In der übersichtlichen Ausbreitung der einschlägigen Literatur liegt der eine wichtige Akzent von Machatscheks Arbeit; der Geograph wird hier mit Nutzen Auskunft holen.

Der Hauptakzent von Machatschexs Werk liegt aber auf der morphotektonischen Beschreibung des Reliefs. Da bewundern wir die ausgedehnte Belesenheit des Autors und die kritisch sichtende Darstellung eines an sich oft recht abstrakten Stoffes. Hier gilt es, das Zusammenspiel von endogenen und exogenen Vorgängen, von Hebungen und Abtragungen, von Senkungen und Auffüllungen, zu erfassen und zeitlich einzuordnen. Es sind vor allem Einebnungsflächen, die eine mehr oder weniger sichere Datierung der einzelnen Phasen erlauben. Маснатsснек verschweigt nicht die Schwierigkeiten der Deutung, die sich aus unsern vielerorts noch mangelhaften Kenntnissen ergeben. Es zeigt sich auch, daß bei klẹnräumigen Studien die chronologische Bedeutung mancher zufälligen und vieldeutigen Kleinform überschätzt und ein kleinliches System errichtet wird, das sich kaum für das betreffende Gebiet, sicher aber nicht für Nachbargebiete eignet. MaChatscheks kritische Bemerkungen zeigen, daß die übertriebene Verfeinerung der Methode nicht nur in den Alpen zu ungehemmten Spekulationen führt $(\star$ Weitere Versuche, eine vielgliedrige Folge von Eintiefungsund Verebnungsphasen aufzustellen [25, 22 oder 19 Phasen] sind als unkritisch abzulehnen.*), sondern in allen bis zum Kleinraum durchforschten Gebieten. (Großbritannien : * Stockwerkflächen ... ohne daß bisher über ihre $Z$ ahl und auch über ihre Entstehungsweise ein endgültiges Urteil oder ihre Parallelisierung über große Käume möglich wäre .... Appalachen: ‘... Bemühungen um Aufstellung möglichst vieler Niveaus...*). Diese und viele andere Beispiele zeigen, daß die überspitzte Verfeinerung der Phasenchronologie manche spekulative Scheinblüte hervorgebracht hat.

Machatscheks Werk vermittelt einen weiten Blick auf das Gebiet der Erforschung des Reliefs, und wir freuen uns, da $\beta$ es, nachdem die erste Auflage durch Einwirkungen des Krieges zum größten Teil zerstört wurde, nun wieder als ein Markstein einer ganzen Forschergeneration in zweiter Auflage neu erstehen konnte. Es bietet aber, wie jedes Standardwerk, nicht nur einen Rück- und Überblick, sondern ermöglicht uns auch einen Ausblick mit weiten Perspektiven auf noch unerschlossene Forschungsgebiete des Reliefs der Erde.

\section{DIE NEUEN OBERSTUFENATLANTEN DES GEOGRAPHISCHEN INSTITUTES ED. HÖLZEL IN WIEN}

\section{EduARD ImHoF}

Seit 1951 erschienen in rascher Folge neue, durch das Geographische Institut Ed. Hölzel in Wien erstellte Oberstufenatlanten für Österreich, Frankreich und Deutschland.

Es sind dies:

1. der e Osterreichische Mittelschulatlas , bearbeitet von Hass StanaR und herausgegeben vom Verlag Ed. Hölzel, Wien 1951 (eine Neubearbeitung des Kozenn-Atlasses).

2. der - Nouvel Atlas Général , bearbeitet von Pierre Serryx, René Blasselle und Marc Bonnet, herausgegeben von Bordas, Paris 1953.

3. der -Atlas zur Erdkunde s, bearbeitet von Hermans LautensaCH, herausgegeben von der Keyserschen Verlagsbuchhandlung, Heidelberg, erste Auflage 1954, zweite Auflage 1955.

Diese drei Atlanten stimmen sowohl im Umfang (146-148 Kartentafeln), Buchformat $(22 \times 30,5 \mathrm{~cm})$, wie auch im graphischen Charakter der Karten miteinander überein. Und nicht nur das, mehr als die Hälfte der Blätter ist, abgesehen von Korrekturen und Nachträgen und abgesehen von der ins Französische übersetzten Beschriftung des «Bordas》, in diesen Atlanten identisch. Dies ergibt sich aus ihrem gemeinsamen Ursprung, denn nicht nur die Reproduktion der Karten, sondern auch ein GroBteil ihrer inhaltlichen Bearbeitung und Zeichnung wurde in Wien durch das Geographische Institut Ed. Hölzel besorgt. Der Grundstock der Karten erschien erstmals im Österreichischen Mittelschulatlas 1951. Etwa 5o Kartentafeln zur Landeskunde Österreichs wurden jedoch für die französische Ausgabe durch Karten französischer Gebiete, für die deutsche Ausgabe durch solche von Deutschland ersetzt. Eine entsprechende Umformung für einen demnächst erscheinenden englischen Atlas ist in Arbeit.

Es wäre nicht richtig, solche Export-Erfolge der Wiener Schulkartographie nur der Geschäftstüchtigkeit des Hölzel-Verlages zuschreiben zu wollen. Inhalt und Form der Atlanten werben für sich. Die Buchformate sind kleiner und handlicher als diejenigen der meisten bisherigen Oberstufenatlanten, auch kleiner als das Format unseres Schweizerischen Mittelschulatlasses. Dies erleichtert ihren Absatz, wird aber andererseits durch Mängel erkauft, auf die wir unten zurückkommen werden. Was diese neuen Wiener Atlanten besonders anziehend 